\title{
GROUPS WITH PRESCRIBED QUOTIENT GROUPS AND ASSOCIATED MODULE THEORY
}




\section{SERIES IN ALGEBRA}

Editors: J. M. Howie, D. J. Robinson, W. D. Munn

Vol. 1: Infinite Groups and Group Rings ed. J. M. Corson et al.

Vol. 2: Sylow Theory, Formations and Fitting Classes in Locally Finite Groups M. Dixon

Vol. 3: Finite Semigroups and Universal Algebra J. Almeida

Vol. 4: Generalizations of Steinberg Groups T. A. Fournelle and K. W. Weston

Vol. 5: Semirings - Algebraic Theory and Applications in Computer Science $U$. Hebisch and H. J. Weinert

Vol. 6: Semigroups of Matrices J. Okninski

Vol. 7: Partially Ordered Groups A. M. W. Glass

Vol. 8: Groups with Prescribed Quotient Groups and Associated Module Theory

L. Kurdachenko, J. Otal and I. Subbotin

Vol. 9: Ring Constructions and Applications A. V. Kelarev 


\section{Published by}

World Scientific Publishing Co. Pte. Ltd.

P O Box 128, Farrer Road, Singapore 912805

USA office: Suite 1B, 1060 Main Street, River Edge, NJ 07661

UK office: 57 Shelton Street, Covent Garden, London WC2H 9HE

\section{British Library Cataloguing-in-Publication Data \\ A catalogue record for this book is available from the British Library.}

\section{GROUPS WITH PRESCRIBED QUOTIENT GROUPS AND ASSOCIATED MODULE THEORY}

Copyright $\odot 2002$ by World Scientific Publishing Co. Pte. Ltd.

All rights reserved. This book, or parts thereof, may not be reproduced in any form or by any means, electronic or mechanical, including photocopying, recording or any information storage and retrieval system now known or to be invented, without written permission from the Publisher.

For photocopying of material in this volume, please pay a copying fee through the Copyright Clearance Center, Inc., 222 Rosewood Drive, Danvers, MA 01923, USA. In this case permission to photocopy is not required from the publisher.

ISBN $981-02-4783-4$

Printed in Singapore by Uto-Print 


\section{To Tamara, Marisol, and Milla}


This page is intentionally left blank 


\section{Preface}

One of the most effective ways to study groups is to evaluate the weight different related subjects have on them. Examples would include considering its subgroups or specific systems of these subgroups (such as normal, subnormal, almost normal, permutable, pronormal, and abnormal subgroups), special subgroups (such as centralizers, normalizers, permutizers), different conjugate classes, groups of automorphisms, rings of endomorphisms of abelian groups, and so on.

Undoubtedly, different natural families of factor-groups (quotients) have an important role in this study. For example, the role of the family of finite factor-groups in different algorithmic problems of finitely presented groups (see, for example, [MA, KM, RRN]), in the theory of the varieties of groups [NH], is well known. The same family plays a relevant role in the study of polycyclic groups. The first result, which illustrated the direct influence of the system of all finite factor-groups on the structure of polycyclic groups, was the following Hirsch's theorem: if every finite factor-group of a polycyclic group $G$ is nilpotent, then $G$ is nilpotent [HIK]. The further investigation of the influence of the system of all finite factor-groups on the structure of polycyclic groups has been developed in different ways and brought many interesting and important results (see, for example, [GPS 1, GPS 2, GS 1, GS 2, GS 3, PP 1, PP 2] and also the book [SD 2]). It has naturally initiated the interest in the study of such influence on the structure of the finitely generated soluble groups. D. J. S. Robinson has obtained a significant amplification of Hirsch's theorem. He proved the following classical result: if every finite factor-group of a finitely generated soluble (even hyperabelian) group $G$ is nilpotent, then $G$ is nilpotent [RD 8]. A similar result has been obtained by D. J. S. Robinson for another class of soluble groups: for residually finite minimax soluble groups [RD 4]. In this association it is important to note that a finitely generated soluble group of finite abelian section rank is minimax (D. J. S. Robinson [RD 18]). Following the result of A. Mann and D. Segal [MS, Theorem A], finitely generated soluble-by-finite minimax groups can be characterized thorough the family of all finite factor-groups in the following 
way: a finitely generated residually finite group $G$ is minimax and soluble-by-finite if and only if there is a number $r$ such that every finite factor-group of $G$ has a (special) rank at most r. Note that D. Segal [SD 3] has considered the influence of some finite factor-groups on the structure of residually finite soluble groups (see, also [DSMS]). Observe also, that research related to different generalizations of this Robinson's theorem, have been launched in the papers [SD 1, ZD 4, BJR 1, BJR 2, BJS]. It is important to admit that this theorem cannot be extended to the property of supersolubility. In other words, there exists a finitely generated soluble group, every finite factor-group of which is supersoluble, but the group itself is not supersoluble (D. Segal [SD 1]).

We also would like to point out the role of the factor-groups by some special subgroups. The Frattini subgroup Fratt $(G)$ of a group $G$ is one of the most influential in this direction. If the Frattini factor-group $G / F r a t t(G)$ of a finitely generated soluble group $G$ is finite, supersoluble or polycyclic, then so is $G$ [LJ 1 , LJ 2, LJ 3].

A significant number of investigations in Group Theory is connected with the consideration of factor-groups by centralizers of different $G$ - invariant subsets.

Let $G$ be a group and $S$ its $G$ - invariant subset (that is $g^{x} \in S$ for every $g \in S$ and every $x \in G$ ). Then $C_{G}(S)$ is a normal subgroup of $G$. The factor-group $G / C G(S)=\operatorname{Coc}_{G}(S)$ is called the cocentralizer of the set $S$ in the group $G$. The group $\operatorname{Coc}_{G}(S)$ is isomorphic with some subgroup of $\left.A u t(<S\rangle^{G}\right)$. The influence of the cocentralizers of many objects connected to a group on the structure of the group is a subject of study in many domains of the Group Theory. Finite Group Theory developed many examples where cocentralizers of chief factors play a great role. We can see it particularly in Formations Theory (note that the local formations are defined by restrictions on cocentralizers of chief factors). Classes of infinite groups, which arise by restriction on cocentralizers of conjugacy classes, are studied.

Let $\mathcal{X}$ be a class of groups. A group $G$ is called an $\mathcal{X}$ - group if $\operatorname{Coc}_{G}\left(g^{G}\right) \in X$ for each $g \in G$.

If $\mathcal{X}=\mathcal{F}$ is the class of all finite groups, then we obtain the well-known class of $F C$ - groups. The class of $F C$ - groups is an extension of the class $\mathcal{F}$ of all finite groups and of the class $\mathcal{A}$ of all abelian groups as well ( $\mathcal{A}$ is exactly the class of $I C$ - groups where $I$ is the class of all identity groups). Therefore they inhered many properties of both of these classes. Currently, the theory of $F C$ - groups forms a well developed topic and represents one of the main branches of the Infinite Groups Theory ( see the books [RD 9, GY, CS 2, TM 1] and the updated survey [TM 2]). B. H. Neumann [NB 1] considered an interesting subclass of $F C$ - groups. He studied the groups with the following properties: $\left|G: C_{G}(x)\right| \leq n$ (or $\left|\operatorname{Coc}_{G}(x G)\right| \leq n$ !) for all elements $x \in G$. These groups are called $B F C$ - groups. B. H. Neumann has proved that $B F C$ - groups are precisely the groups with finite derived subgroups (or finite-by-abelian groups). In connection with this result the following problem arises. Let $G$ be a group, put $b(G)=\max \left\{\left|G: C_{G}(g)\right| \mid\right.$ $g \in G\}$ and suppose that $b(G)$ is finite. Are the numbers $b(G)$ and $|[G, G]|$ in relation? A whole series of interesting articles have been dedicated to defining of 
this connection (see [BI, CM, MI, NP, NPV-L, V-L, SS, WI 1, WI 2].

If $\mathcal{X}=\mathcal{C}$ is the class of all Chernikov groups, then we obtain the class of groups with Chernikov conjugacy classes or $C C$ - groups. Ya. D. Polovitsky in [PY] introduced this class and obtained some initial results. Although $C C$ - groups are not investigated as far as $F C$ - groups, they are the subject of many recent papers (see [AO, FdeGT 2, GO 1, GO 2, OP 1, OP 2, OPT] and others).

The study of $\mathcal{X} C$ - groups for other important classes $\mathcal{X}$ has only recently begun (see [FdeGK 1, FdeGT 1, KL 4, KL 6, KSU 1]).

The widest family of the factor-groups of a group $G$ is the family of all its proper factor-groups. If $H$ is a non-identity normal subgroup of $G$, then $G / H$ is said to be a proper factor-group. Let $\mathcal{X}$ be a class of groups. A group $G$ is called a just non- $\mathcal{X}$-group if every proper factor-group of $G$ belongs to $\mathcal{X}$, but $G \notin \mathcal{X}$. This book is entirely devoted to just non- $\mathcal{X}$-groups, and describes techniques and methods of study in detail. It also presents the most recently developed results of the theory for several most important choices of the class $\mathcal{X}$.

The structure of the just non- $\mathcal{X}$-groups has been already studied for many natural classes of groups $\mathcal{X}$. The first important research here is due to $\mathrm{M}$. $\mathrm{F}$. Newman [NM 1, NM 2], who considered the just non-abelian groups. The second crucial step in this direction was the consideration of just infinite groups (more properly, just non-finite groups), which has been realized by D. McCarthy [McC 1, McC 2] and J. S. Wilson [WJ 2]. Just infinite groups, which are near to soluble groups have been considered in [ $\mathrm{McC} 1, \mathrm{McC} 2$ ]. In [WJ 2], J. S. Wilson considered a general situation. The characteristics properties of these groups obtained in this paper could be used for investigation of the structure of some interesting examples of finitely generated periodic groups such as the Grigorchuk group or the Gupta - Sidki group, the study of which is very intensive recently.

The next step is connected to the notion of a $T$ - group. The class of the groups in which normality is a transitive relation ( $T$-groups) has been studied well by now. These groups and some of their generalizations have been investigated by many authors ([AI, AK, BT, GW, RD 1, RD 5, MF 1, MF 2, ZG] and many others). The most valuable results about $T$-groups have been obtained by D. J. S. Robinson in [RD 1]. This work, together with the works mentioned above, established the wide variety of interesting and important results, placing $T$-groups and their generalizations in the main stream of study of the normal structure of groups (see, for example, the survey [RD 24].) In [RD 11] D. J. S. Robinson has described soluble just non- $T$-groups. This class of groups had some peculiar properties. If just non-abelian groups were monolithic, and the infinite groups were non-monolithic, then both of these situations meet combined already in the study of just non-T-groups.

All studies that followed were connected with distinct extensions of the class $\mathcal{A}$ of all abelian groups, and of the class $\mathcal{F}$ of all finite groups as well. Thus J. R. J. Groves [GJ], and D. J. S. Robinson and J. S. Wilson [RW] considered soluble groups with polycyclic or supersoluble proper factor-groups. The paper [RW], which is very rich with new ideas, constructions and results, took a remarkable place in the theory of just non- $\mathcal{X}$-groups, and determined in many different ways 
the further development of this theory. In particular, it showed the meaning of the module theoretical methods for the study of the just non- $\mathcal{X}$-groups. The key role of just infinite modules has been demonstrated in this paper.

Dual to the class of polycyclic groups is the class of Chernikov groups (the polycyclic groups can be characterized as soluble groups with maximal condition; the Chernikov groups can be characterized as soluble-by-finite groups with minimal condition). The groups with Chernikov proper factor-groups have been considered by S. Franciosi and F. de Giovanni [FdeG 1], and also by L. A. Kurdachenko, V. E. Goretsky and V. V. Pylaev [KGP]. In the article [KSU 4] L. A. Kurdachenko and I. Ya. Subbotin investigated the more general case when all proper factor-groups are hyperfinite (obviously all Chernikov groups are hyperfinite).

The next important step was made in the paper of D. J. S. Robinson and Z. Zheng [RZ], in which they described just non-(central-by-finite)-groups and just non-(finite-by- abelian)-groups (or just non-BFC-groups). Both classes, central-by-finite groups and finite-by-abelian ( $B F C$ - groups), are extensions of finite groups and abelian groups. On the other hand, both of these classes are proper subclasses of $F C$ - groups. Therefore, the study of just non- $F C$-groups (S. Franciosi, F. de Giovanni, L. A. Kurdachenko [FdeGK 3]) seems very natural in this direction. The papers of L. A. Kurdachenko and J. Otal [KO 1, $\mathrm{KO} 2, \mathrm{KO} 3$ ], in which just non- $C C$-groups have been studied, followed after. The other natural restrictions of the class $\mathcal{A}$ are the classes $N_{c}$, the class of nilpotent groups of nilpotence class $\leq c$, and $N$, the class of all nilpotent groups. S. Franciosi and $\mathrm{F}$. de Giovanni [FdeG 2] considered the just non- $N_{c}$-groups and just non-nilpotent groups. A slight generalization of this situation was considered by Z. Zhang [ZZ]. L. A. Kurdachenko and I. Ya. Subbotin in their paper [KSU 3] initiated the important investigation of just non-hypercentral groups.

The main goal of this book is to provide the systematic and complete exposition of the main results of the theory of just non- $\mathcal{X}$-groups. We expose the material in a way that allows us to describe the oldest and newest results from a common point of view. Such an approach is determined by the following circumstances, which in general define the main content of the book.

First, let's talk about some important restrictions. Clearly, if $G$ is a simple group, then $\mathrm{G}$ is a just non-X-group for each class of groups $\mathcal{X}$. This means that the study of just non- $\mathcal{X}$-groups requires some additional restrictions allowing us to avoid this situation. Therefore, as in most papers about just non- $\mathcal{X}$-groups, we will only consider such groups $G$ whose Fitting subgroup Fitt $(G)$ is non-identity. We recall that $F i t t(G)$ is the subgroup generated by all normal nilpotent subgroups of $G$; so the condition $\operatorname{Fitt}(G) \neq<1>$ is equivalent to the condition for $G$ to include a non-identity normal abelian subgroup.

Also, this approach is determined by possibilities to apply Module Theory. Let $G$ be a just non- $\mathcal{X}$-group. For many important classes $\mathcal{X}$ the investigation of $G$ is very often split into two parts according to the following two alternatives: $F C(G) \neq<1>$ and $F C(G)=\langle 1\rangle$ (here $F(G)$ is the $F C$-center of a group $G$ ). The study of the first case, when $F C(G) \neq<1>$, usually is realized with no 
problems. Therefore, the case $F C(G)=\langle 1\rangle$ is the most interesting one. For almost all considered classes $\mathcal{X}$, the subgroup $\operatorname{Fitt}(G)=A$ is abelian; moreover, it is either an elementary abelian $p$ - subgroup for some prime $p$ or an abelian torsion-free subgroup. Then the group $H=G / A$ naturally acts on $A$ by conjugation and this action gives raise to $A$ into a $\mathbb{Z} H$ - module. This shows that the study of just non- $\mathcal{X}$-groups requires the study of some types of modules over the group rings.

Let $\mathcal{P}$ be a family of all non-identity $G$ - invariant subgroups of $A, M=\cap \mathcal{P}$. Obviously $M$ can be identity or not, and the study of $G$ is very dependent on it. Therefore, there is a need to split the study into two cases:

$$
\begin{gathered}
\text { (M) } M \neq<1>, \\
\text { and } \\
\text { (N-M) } M=<1>\text {. }
\end{gathered}
$$

In the case (M) the subgroup $M$ is the monolith of the group $G$; in particular, $M$ is a simple $\mathbb{Z} H$ - module. For many important classes $\mathcal{X}$ in the monolithic case $A=M$, in other words, $A$ is a simple $\mathbb{Z} H$ - module. Thus, the first important type of modules that arises in research of just non- $\mathcal{X}$-groups is the class of simple modules. This type of module has arisen already in the first paper dedicated to this theme, in the paper of M. F. Newman [NM 1]. Hence, one of our tasks will be the study of simple modules over $\mathcal{X}$-groups, which will be carried out in several chapters of this book. These modules play a key role not only in the study of the just non- $\mathcal{X}$-groups but also in other issues of Group Theory (see, for example, the survey $[\mathrm{KZK}]$ ). Other related questions will also be considered.

Recall that an infinite module $A$ is said to be just infinite, if it is non-monolithic and every one of its proper submodules has finite index. In the case (N-M) (the non-monolithic case) just infinite modules play a very important role. These modules have been introduced in the important paper of D. J. S. Robinson and J. $\mathrm{S}$. Wilson [RW]. Further research has shown that these modules are crucially important to the study of just non- $\mathcal{X}$-groups and for many different important classes $\mathcal{X}$ of groups.

The first modular part of this book is completely determined by the arguments above. It will be devoted to considerations of simple and just infinite modules over some important types of groups. Moreover, we want to consider modules over the ring $R H$ where $R$ is some "good" extension of the both rings $\mathbb{Z}$ and $\mathbb{F}_{p}\langle g\rangle$ where $\langle g\rangle$ is an infinite cyclic group, which are especially important for group-theoretic applications. This extension was carried out by considering a Dedekind domain as such a ring. We recall that a Dedekind domain is a noetherian integrally closed domain, in which every non-zero prime ideal is maximal.

When we are thinking about extending the theory of abelian groups on the modules, we have to bear in mind that these extensions involve the coefficients ring and occur step by step, from result to result. Many results that are valid for the ring $\mathbb{Z}$ are also valid for many of its wide ring extensions This is a good justification of the introduction of the most important classes of commutative 
rings: principal ideal domains, Dedekind domains, valuation domains, integral domains and so on (at this point, the comments from the chapters of [FL 1,FL 2] and [FLS] are useful). Many years of experience proved that Dedekind domains have appeared in the most achieved generalizations of the Abelian Group theory. To be more specific, they form the widest class of integral domains, to which the main results from Abelian Group Theory can be extended; moreover, these analogies produce new characterizations of this class. Therefore, in the sequel, we will consider modules over a group ring $D G$, where $D$ is a Dedekind domain.

The module part is key part of the study of all such types of just non- $\mathcal{X}$-groups that are considered in this book. However, this investigations are not exhausted by the research of corresponding modules. Along with some general elements of structure, each class of groups considered in the book has its own specific individual characteristics and, therefore, requires an individual consideration.

This book includes a relatively large theoretical part of the group, in which we try to expose most important known results from our point of view in this area. The mentioned restrictions and the limited size of the book do not allow us to provide all existing information, however, we try to reflect the information as completely as possible in the bibliography.

The authors want to express their deepest gratitude to D. J. S. Robinson, whose results and methods exercised significant influence on the authors' interest in the theme. They also treasure very much his extremely valuable comments and his inspiration during the process of preparing this book.

Also they would like to mention the names of the prominent scholars, whose personal influences, tests, and encouragements played a key role in the formation of the authors as mathematicians. Unfortunately, these wonderful people passed away some time ago, but the memory of them still supports the authors throughout the hard way of mathematical research. They are S. N. Chernikov, D. I. Zaitsev and Z. I. Borevich.

We would like to thank World Scientific Publishing Co, Pte Ltd for the great opportunity to publish this book in its famous Algebra Series. We really treasure personal help and encouragement, which came from the Commissioner Editor A. Doyle and from the Scientific Editor J. T. Lu.

Scott McClintock and Karen Offitzer helped us tremendously with the editing.

This work would never have been completed without the great support of National University, U. S. A. and Zaragoza University, Spain. The authors are very thankful for that. 


\section{Contents}

Preface

vii

Notation

\section{Simple Modules}

1. On Annihilators of Modules 3

2. The Structure of Simple Modules over Abelian Groups 17

3. The Structure of Simple Modules over Some Generalization of Abelian Groups

4. Complements of Simple Submodules

\section{Just Infinite Modules}

5. Some Results on Modules over Dedekind Domains 47

6. Just Infinite Modules over FC-Hypercentral Groups 61

7. Just Infinite Modules over Groups of Finite 0-Rank 77

8. Just Infinite Modules over Polycyclic-by-Finite Groups 91

9. Co-Layer-Finite Modules over Dedekind Domains 101

\section{Just Non- $\chi$-Groups}

10. The Fitting Subgroup of Some Just Non- $\chi$-Groups 109

11. Just Non-Abelain Groups 115

12. Just Non-Hypercentral Groups and Just Non-Hypercentral Modules 121

13. Groups with Many Nilpotent Factor-Groups 131

14. Groups with Proper Periodic Factor-Groups 143

15. Just Non-(Polycyclic-by-Finite) Groups 155

16. Just Non- $C C$-Groups and Related Classes 165

17. Groups Whose Proper Factor-Groups Have a Transitive Normality Relation

Bibliography

Author Index $\quad 221$

Subject Index 
This page is intentionally left blank 


\section{Notation}

$$
\begin{aligned}
& A n n_{R}(a) \quad 3 \\
& A n n_{R}(A) \quad 3 \\
& t_{R}(A) \quad 3 \\
& S p e c(D) \quad 4 \\
& \prod_{D}(a) \quad 4 \\
& \Pi_{D}(A) \quad 4 \\
& A_{I} \quad 4 \\
& \Omega_{I, n}(A) \quad 4 \\
& A(D, \pi) \quad 5 \\
& r_{R}(A) \quad 6 \\
& r_{0}(G) \quad 11 \\
& \mathcal{X} C(G) \quad 28 \\
& \mathcal{Q X} \quad 28 \\
& \mathcal{R}_{0} \mathcal{X} \quad 28 \\
& \mathcal{X C} C_{\infty}(G) \quad 28 \\
& F C(G) \quad 28 \\
& F C_{\infty}(G) \quad 28 \\
& Q s o c(G) \quad 32 \\
& \operatorname{Soc} c_{a b}(G) \quad 32 \\
& \mathbf{S}_{n} \mathcal{X} \quad 69 \\
& \mathbf{P X} \quad 69 \\
& \zeta_{R G}(A) \quad 84 \\
& \mu(G) \quad 109
\end{aligned}
$$




$$
\begin{array}{ll}
\mu_{R G}(A) & 122 \\
\rho_{R G}(A) & 122
\end{array}
$$

